\title{
FINITENESS AT INFINITY
}

\author{
by P. A. FIRBY† \\ (Received 13th June 1970)
}

\section{Introduction and preliminaries}

If $X$ is a Tychonoff topological space, and if $\beta X$ is the Stone-Cech compactification of $X$, then $\beta X \backslash X$ will denote the complement of $X$ in $\beta X$. If $A$ is a subset of $X$, then $\operatorname{cl}[A: X]$ will denote the closure of $A$ in $X$, and int $[A: X]$ will denote the interior of $A$ in $X$. In Isbell ((3), p. 119) a property of $\beta X\rceil X$ is called a property which $X$ has at infinity, and it is the aim of this paper to give necessary and sufficient conditions for $X$ to be finite at infinity. Since $\beta X$ is $T_{1}$ we can say that if $X$ is finite at infinity, then $\beta X \backslash X$ is closed in $\beta X$. So we lose nothing by restricting our attention to locally compact, Tychonoff spaces, and for the remainder of the paper $X$ will denote such a space.

The closed sets $A$ and $B$ are said to be completely separated if and only if there exists a bounded, continuous, real-valued function $f$ on $X$ taking the value 0 on $A$ and the value 1 on $B$. From the description of $\beta X$ in terms of such functions we see that $A$ and $B$ are completely separated in $X$ if and only if $\mathrm{cl}[A: \beta X] \cap \mathrm{cl}[B: \beta X]=\varnothing$.

Unless otherwise stated, uniformities on $X$ are assumed to be compatible with the topology of $X$.

The following result is due to Doss ((1), p. 20): there is just one uniform structure on $X$ if and only if, of any two completely separated closed sets in $X$, one is compact.

Let $C^{*}(X)$ be the algebra of bounded, continuous real-valued functions on $X$ with the topology of uniform convergence on the space $X$. Let $A(X)$ be the subalgebra of $C^{*}(X)$ consisting of those functions each of which is constant on the complement of some compact subset of $X$. Gál ((2), p. 1053) has proved: there is just one uniform structure on $X$ if and only if $A(X)$ is dense in $C^{*}(X)$.

Now there is just one uniform structure on $X$ if and only if $\beta X$ is the Alexandroff compactification of $X$, so we can write the preceding results in the form

The following statements are equivalent:

(a) $|\beta X \backslash X|=1$.

(b) Of any two closed, completely separated sets in $X$, at least one is compact.

(c) $A(X)$ is dense in $C^{*}(X)$.

In this form, we extend these results in the next three sections.

† Research supported by S.R.C. grant. 
2. Conditions on $X$ for $X$ to have power $n$ at infinity

Lemma 2.1. If $|\beta X \backslash X|=m$ ( $m$ any cardinal), then any collection $C$ of closed sets in $X$, completely separated in pairs and such that $|C|>m$, contains a compact member.

Proof. Suppose $C=\left\{C_{\lambda}: \lambda \in \Lambda\right\}$ where $|\Lambda|>m$. Then

$$
\left\{\operatorname{cl}\left[C_{\lambda}: \beta X\right]: \lambda \in \Lambda\right\}
$$

is a collection of sets disjoint in pairs, and so no element of $\beta X \backslash X$ belongs to more than one member of this collection. Since $|\Lambda|>|\beta X| X \mid$ there exists $\mu \in \Lambda$ such that $\mathrm{cl}\left[C_{\mu}: \beta X\right]=C_{\mu}$.

Then $C_{\mu}$ is compact as required.

We can now give the required condition on $X$.

Theorem 2.2. $|\beta X \backslash X| \leqq n$ ( $n$ finite) if and only if, of any $n+1$ closed sets in $X$, completely separated in pairs, at least one is compact.

Proof. Suppose $|\beta X| X \mid>n$ and choose $n+1$ distinct points $a_{1}, \ldots, a_{n+1}$ in $\beta X \backslash X$. Let $U_{1}, \ldots, U_{n+1}$ be neighbourhoods of $a_{1}, \ldots, a_{n+1}$ respectively. Since $\beta X$ is $T_{2}$ and compact, we can assume these neighbourhoods to be compact and disjoint in pairs. Now let $B_{i}=U_{i} \cap X \neq \varnothing$, so that $\operatorname{cl}\left[B_{i}: \beta X\right] \subseteq U_{i}$ $(i=1, \ldots, n+1)$. Thus the $B_{i}$ 's are completely separated in pairs and each $B_{i}$ is closed in $X$. On the other hand, each $B_{i}$ fails to be compact, since it contains a net converging to $a_{i} \in \beta X \backslash B_{i}(i=1, \ldots, n+1)$.

The reverse implication follows from Lemma 2.1 .

Note that the inequality can be removed from the statement of Theorem $\mathbf{2 . 2}$ to give:

Theorem 2.2'. $|\beta X| X \mid=n$ ( $n$ finite) if and only if there exist $n$ closed, noncompact sets in $X$ which are completely separated in pairs, but no such collection of $n+1$ sets exists.

\section{Conditions on $C^{*}(X)$ for $X$ to have power $n$ at infinity}

Define $B_{n}(X)$ to be the subset of $C^{*}(X)$ consisting of those functions which take at most $n$ values ( $n$ finite) on the complement of a compact subset of $X$.

Theorem 3.1. If $|\beta X \backslash X|=n$ (n finite), then $B_{n}(X)$ is uniformly dense in $C^{*}(X)$.

Proof. Let $f: X \rightarrow[0,1]$ be continuous and denote by $f$ the continuous extension of $f$ to $\beta X$. Suppose $\beta X \backslash X=\left\{a_{1}, \ldots, a_{n}\right\}$ and $f\left(a_{i}\right)=b_{i}(i=1, \ldots, n)$. If necessary, relabel $b_{1}, \ldots, b_{n}$ as the distinct numbers $c_{1}, \ldots, c_{m}$ with $m \leqq n$.

Let $\varepsilon>0$ be given, and let $\delta$ be equal to the smallest member of

$$
\left\{\{\varepsilon\} \cup\left\{\left|c_{i}-c_{j}\right|: i \neq j\right\}\right\} \text {. }
$$


Put $U_{i}=\left\{x \in \beta X:\left|\vec{f}(x)-c_{i}\right|<\frac{1}{2} \delta\right\}(i=1,2, \ldots, m)$. For each $i \in\{1, \ldots, m\}$ choose a closed neighbourhood (in $\beta X$ ) $V_{i}$ of $\left\{a_{j}: \bar{f}\left(a_{j}\right)=c_{i}, j=1, \ldots, n\right\}$ such that $V_{i} \subseteq U_{i}$.

Since $\beta X$ is normal, given $i \in\{1, \ldots, m\}$ there exists $\bar{g}_{i}: \beta X \rightarrow[0,1]$ such that $\bar{g}_{i}$ is continuous and

$$
\begin{cases}\bar{g}_{i}(x)=0 & \left(x \in V_{i}\right) \\ \bar{g}_{i}(x)=1 & \left(x \in \beta X \mid U_{i}\right)\end{cases}
$$

Denote by $g_{i}$ the restriction of $\bar{g}_{i}$ to $X(i=1, \ldots, m)$ and define

$$
\begin{aligned}
h_{1} & =c_{1}+\left(f-c_{1}\right) g_{1}, \\
h_{i} & =c_{i}+\left(h_{i-1}-c_{i}\right) g_{i} \quad(i=2, \ldots, m) .
\end{aligned}
$$

Then for each $i \in\{1, \ldots, m\}$ the following properties $(a),(b),(c)$ hold:

(a) $h_{i}: X \rightarrow[0,1]$ is continuous.

Clearly, $h_{i}$ is continuous. Also, $h_{i}(x)=c_{i}\left[1-g_{i}(x)\right]+h_{i-1}(x) \cdot g_{i}(x)$, which is a number between $c_{i}$ and $h_{i-1}(x)$. A simple finite induction argument shows that $h_{i}(x) \in[0,1](x \in X, i \in\{1, \ldots, m\})$, establishing $(a)$.

(b) $h_{i}(x)=c_{j}\left(x \in X \cap \operatorname{nint}\left[V_{j}: \beta X\right]\right)$ for each $j \in\{1, \ldots, i\}$.

To prove this let $W_{j}=X \operatorname{nint}\left[V_{j}: \beta X\right](j \in\{1, \ldots, m\})$. Clearly, $h_{1}(x)=c_{1}$ $\left(x \in W_{1}\right)$. Suppose then that $h_{k}(x)=c_{j}\left(x \in W_{j}, 1 \leqq j \leqq k\right)$ for each $k \in\{1, \ldots, i-1\}$. Consider $h_{i}(x)=c_{i}+\left(h_{i-1}(x)-c_{i}\right) g_{i}(x)$.

Then $g_{i}(x)=1\left(x \in W_{j}, 1 \leqq j<i\right)$, and so $h_{i}(x)=h_{i-1}(x)=c_{j}\left(x \in W_{j}\right.$, $1 \leqq j<i)$. Also $g_{i}(x)=0\left(x \in W_{i}\right)$, whence $h_{i}(x)=c_{i}\left(x \in W_{i}\right)$. This completes the proof of $(b)$.

$$
h_{i}(x)=f(x)\left(x \in X \backslash\left[X \cap \bigcup_{j=1}^{i} U_{j}\right]\right) .
$$

The proof is straightforward.

In particular, $(a),(b)$ and $(c)$ hold for $h_{m}$.

We now show that $\left|h_{m}(x)-f(x)\right|<\varepsilon(x \in X)$. In fact, from $(b)$ and $(c)$, the inequality need only be proved for $x$ in $T_{i}=X \cap\left(U_{i} \backslash V_{i}\right)(i \in\{1, \ldots, m\})$. Clearly,

$$
\left|h_{1}(x)-f(x)\right| \leqq \frac{1}{2} \delta<\varepsilon \quad\left(x \in T_{1}\right) .
$$

Suppose then that $\left|h_{i}(x)-f(x)\right|<\varepsilon\left(x \in T_{j}, j \in\{1, \ldots, i\}\right)$ for each $i=1, \ldots, k-1$. Consider $h_{k}$ on $T_{j}(j \in\{1, \ldots, k-1\})$. Since $j<k, g_{k}(x)=1\left(x \in T_{j}\right)$. Thus $h_{k}(x)=c_{k}+\left(h_{k-1}(x)-c_{k}\right) g_{k}(x)=h_{k-1}(x)$. Next, on $T_{k}, h_{k-1}(x)=f(x)$, so that

$$
\begin{aligned}
\left|h_{k}(x)-f(x)\right| & =\left|c_{k}+\left[f(x)-c_{k}\right] g_{k}(x)-f(x)\right| \\
& =\left|c_{k}-f(x)\right| .\left|1-g_{k}(x)\right| \leqq \frac{1}{2} \delta<\varepsilon .
\end{aligned}
$$

By the principle of finite induction, the required inequality follows.

Finally, for each $i \in\{1, \ldots, m\}$, int $\left[V_{i}: \beta X\right]$ is an open neighbourhood of $a_{i}$ in $\beta X$ having empty intersection with $Y=X \backslash\left(\left[\bigcup_{i=1}^{m}\right.\right.$ int $\left.\left.\left[V_{i}: \beta X\right]\right] \cap X\right)$ and 
so $Y$ is compact. But from $(b)$ we see that $h_{m}$ takes $m$ values on $X \backslash Y$, and so

$$
f \in \operatorname{cl}\left[B_{m}(X): C^{*}(X)\right] \subseteq \operatorname{cl}\left[B_{n}(X): C^{*}(X)\right] \text {. }
$$

It follows that $B_{n}(X)$ is uniformly dense in $C^{*}(X)$.

Lemma 3.2. If $B_{n}(X)$ is uniformly dense in $C^{*}(X)$ then $|\beta X \backslash X| \leqq n$.

Proof. Suppose $|\beta X \backslash X|>n$ and let $a_{1}, \ldots, a_{n+1}$ be distinct elements of $\beta X \backslash X$. Choose, for $i=1, \ldots, n+1$, mutually disjoint open neighbourhoods $U_{i}$, in $\beta X$, of $a_{i}$, together with closed neighbourhoods $V_{i}$ of $a_{i}$ such that $V_{i} \subseteq U_{i}$. Since $\beta X$ is normal, given $i \in\{1, \ldots, n+1\}$, there exists a continuous function $f_{i}: \beta X \rightarrow[0, i]$ such that $f_{i}\left(V_{i}\right)=i$ and $f_{i}\left(\beta X \backslash U_{i}\right)=0$.

Now define $f: \beta X \rightarrow[0, n+1]$ by $f=\sum_{i=1}^{n+1} f_{i}$. Then $f$ is continuous and $f\left(a_{i}\right)=i(i=1, \ldots, n+1)$. Let $h$ denote the restriction of $(n+2)^{-1} f$ to $X$. Then

$$
h(x)=(n+2)^{-1} i \quad\left(x \in X \cap \operatorname{nint}\left[V_{i}: \beta X\right], i=1, \ldots, n+1\right) .
$$

Let $g \in B_{n}(X)$ be such that $\sup \{|h(x)-g(x)|: x \in X\}<\frac{1}{2}(n+2)^{-1}$ and let $K$ be a compact set such that outside $K, g$ assumes at most $n$ distinct values. Since $K$ is compact, for each $i \in\{1, \ldots, n+1\}$ there is a neighbourhood $W_{i}$ of $a_{i}$ in $\beta X$ such that $K \cap W_{i}=\varnothing$. Let $A_{i}=X \cap \operatorname{int}\left[V_{i}: \beta X\right] \cap W_{i}$. Then $A_{i} \neq \varnothing$, and $h(x)=(n+2)^{-1} i\left(x \in A_{i} ; i=1, \ldots, n+1\right)$. Clearly, we cannot have

$$
|h(x)-g(x)|<\frac{1}{2}(n+2)^{-1} \quad\left(x \in \bigcup_{i=1}^{n+1} A_{i}\right)
$$

unless $g$ takes at least $n+1$ distinct values on the complement of $K$. This implies that $B_{n}(X)$ is not uniformly dense in $C^{*}(X)$.

From Theorem 3.1 and Lemma 3.2 we then get

Theorem 3.3. $|\beta X \backslash X| \leqq n$ (n finite) if and only if $B_{n}(X)$ is uniformly dense in $C^{*}(X)$.

Once again we note that the inequality in Theorem 3.3 can be removed to give

Theorem 3.3'. $|\beta X \backslash X|=n$ (n finite) if and only if $B_{n}(X)$ is uniformly dense in $C^{*}(X)$ and $B_{n-1}(X)$ is not.

We note that if $a X$ is a compactification of $X$ (we consider $X$ to be a subspace of any compactification of $X$ ) and if $C_{a}(X)$ denotes the subset of $C^{*}(X)$ consisting of those functions having a continuous extension to $a X$, then the proofs of this section carry through, with slight modifications, to give

Theorem 3.4. $|a X \backslash X|=n$ and $a X$ is the unique (up to equivalence of compactifications) n-point compactification of $X$ if and only if $B_{n}(X)$ is uniformly dense in $C_{o}(X)$ and $B_{n-1}(X)$ is not. 


\section{Conditions on $X$ for $X$ to be finite at infinity}

Theorem 4.1. $X$ is finite at infinity if and only if every infinite collection of closed sets in $X$, completely separated in pairs, contains a compact member.

Proof. Suppose $X$ is infinite at infinity and let $\left(x_{n}\right)$ be an infinite sequence of distinct points of $\beta X \backslash X$. Let $x$ be a cluster point of $\left(x_{n}\right)$ in

$$
\beta X \backslash\left\{x_{n}: n=1,2, \ldots\right\} \text {. }
$$

We define a sequence $\left(y_{n}\right)$ recursively as follows. Put $y_{1}=x_{1}$. Then there exist disjoint closed neighbourhoods $U_{1}, V_{1}$ of $x$ and $y_{1}$ respectively in $\beta X$. Since $x$ is a cluster point of $\left(x_{n}\right)$ there is $x_{n_{2}}$ in $U_{1}$. Set $y_{2}=x_{n_{2}}$. Then choose a closed neighbourhood $V_{2}$ of $y_{2}$ and a closed neighbourhood $U_{2}$ of $x$ such that $V_{2} \cap V_{1}=V_{2} \cap U_{2}=\varnothing$. Now suppose $y_{1}, \ldots, y_{k}$ have been chosen with

$$
y_{i} \in U_{i-1}, V_{i} \cap U_{i}=\varnothing, V_{i} \cap \bigcup_{j=1}^{i-1} V_{j}=\varnothing \quad(i=1, \ldots, k) .
$$

(This is possible since the $V_{i}$ are compact.) Since $x$ is a cluster point of $\left(x_{n}\right)$, there is $x_{n_{k+1}}$ in $U_{k}$. Set $y_{k+1}=x_{n_{k+1}}$. Choose neighbourhoods $V_{k+1}$ of $y_{k+1}$ and $U_{k+1}$ of $x$ such that $V_{k+1} \cap \bigcup_{i=1}^{k} V_{i}=\varnothing$ and $V_{k+1} \cap U_{k+1}=\varnothing$.

This process defines a sequence $\left(V_{k}\right)$ of closed (compact) subsets of $\beta X$ which are mutually disjoint. Hence $\left(X \cap V_{k}\right)$ is a sequence of closed subsets of $X$, completely separated in pairs. Since $y_{k}$ in $V_{k}$ is adherent to $\left(X \cap V_{k}\right)$ in $\beta X$, no set in this collection is compact. The desired implication follows.

On the other hand, if $X$ is finite at infinity, Lemma 2.1 applies, and every infinite collection of closed subsets of $X$, completely separated in pairs, contains a compact member.

From Theorems 4.1 and 2.2 we then get the following result within $X$.

Corollary 4.2. If every infinite collection of closed sets in $X$, completely separated in pairs, contains a compact member, then there exists an integer $n$ such that every collection of $n$ closed sets in $X$, completely separated in pairs, contains a compact member.

Now, using Theorem 4.1 we obtain the following necessary condition for a normal space $X$ to be finite at infinity.

Corollary 4.3. If $X$ is normal, then $X$ finite at infinity implies that $X$ is countably compact.

Proof. Suppose $X$ fails to be countably compact. Then there is a sequence $\left(U_{n}\right)$ of open sets in $X$ such that $U_{n} \subset U_{n+1}(n=1,2, \ldots), \bigcup_{n} U_{n}=X$, and no finite collection of $U_{n}$ 's covers $X$. Then choose $x_{n+1} \in U_{n+1} \mid U_{n}(n=1,2,3, \ldots)$. This defines an infinite sequence $\left(x_{n}\right)$ of distinct points in $X$. For $i=1,2, \ldots$, let $s_{i}=2^{i-1}$ and put $A_{i}=\left\{x_{s_{i}(2 n+1)}: n=0,1,2, \ldots\right\}$. 
Now, if $x \in X \backslash A_{i}$, then for some integer $N, x \in U_{N}$. Then $U_{N}$ is a neighbourhood of $x$ containing only a finite number of members of $A_{i}$, and so, since $X$ is $T_{2}, x \notin \operatorname{cl}\left[A_{i}: X\right]$. It follows that, for each $i, A_{i}$ is closed in $X$.

We see next that the members of $\left\{A_{i}: i=1,2, \ldots\right\}$ are disjoint in pairs, for if $i<j$ and $x_{s_{i}(2 n+1)}=x_{s_{j}(2 m+1)}$ for some $m, n$, then since the members of $\left(x_{n}\right)$ are distinct, $s_{i}(2 n+1)=s_{j}(2 m+1)$ and so $2 n+1=2^{j-i}(2 m+1)$.

So we have an infinite collection of closed sets in $X$, disjoint in pairs. Since $X$ is normal we see from Urysohn's Lemma that the members of this collection are completely separated in pairs, and so from Theorem 4.1 we see that $X$ is infinite at infinity.

Note that if $\Omega_{0}$ denotes the set of all ordinal numbers less than the first uncountable ordinal, with the order topology, then $\beta\left(\Omega_{0}\right)$ is the set of ordinals less than or equal to the first uncountable ordinal i.e. $\left|\beta\left(\Omega_{0}\right)\right| \Omega_{0} \mid=1$. So by taking suitable disjoint unions of the space $\Omega_{0}$ with itself we obtain examples of spaces having any given finite power at infinity.

Note also that $\Omega_{0} \times \Omega_{0}$ is normal, $T_{2}$, countably compact and locally compact, and so, since $\beta\left(\Omega_{0} \times \Omega_{0}\right)=\beta\left(\Omega_{0}\right) \times \beta\left(\Omega_{0}\right)$, we have a counterexample to the converse implication to Corollary 4.3.

Finally, we note that since $\beta X \backslash X$ is finite if and only if $\beta X \backslash X$ is discrete, we can replace " finite at infinity" throughout by " discrete at infinity".

In conclusion I would like to express my gratitude to the referee for his helpful comments, and in particular for his streamlining of my original proofs of Theorems 3.1 and 4.1 and of Lemma 3.2.

\section{REFERENCES}

(1) R. Doss, On uniform spaces with a unique structure, Amer. J. Math. 71 (1949), 19-23.

(2) I. S. GÁL, Uniformisable spaces with a unique structure, Pacific J. Math. 9 (1959), 1053-1060.

(3) J. R. Isbell, Uniform spaces (Mathematical Surveys, No. 12, American Math. Soc., Providence, R.I., 1964).

(4) J. L. Kelley, General Topology (Van Nostrand, London, 1955).

THE UNIVERSITY

SHEFFIELD

ENGLAND 